\title{
Retraction Note to: Analysis of loxoprofen in tablets, patches, and equine urine as tert-butyldimethylsilyl derivative by gas chromatography-mass spectrometry
}

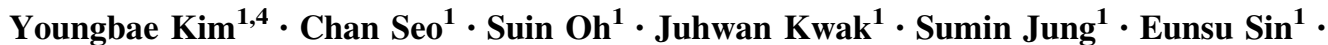 \\ Hyunbin $\mathrm{Kim}^{1}$ • Moongi $\mathrm{Ji}^{1} \cdot$ Hyeon-Seong Lee ${ }^{1} \cdot$ Hyung-Jin Park ${ }^{2} \cdot$ Gwang Lee $^{2}$. \\ Jundong $\mathrm{Yu}^{3} \cdot$ Minsoo Kim $^{4} \cdot$ Wonjae Lee $^{5} \cdot$ Man-Jeong Paik $^{1}$ (D)
}

Published online: 1 February 2019

(c) The Pharmaceutical Society of Korea 2019

Retraction to: Arch. Pharm. Res. (2018) 41:459-466 https://doi.org/10.1007/s12272-018-1023-5

The authors have retracted this article [1] because after publication they became aware that the equine urine samples analysed for loxoprofen in this study were in fact equine plasma samples. Therefore the results and conclusions of this article cannot be relied upon. All authors agree to this retraction.

The original article can be found online at https:// doi.org/10.1007/s12272-018-1023-5.

Wonjae Lee

wlee@chosun.ac.kr

$\triangle$ Man-Jeong Paik

paik815@sunchon.ac.kr

1 College of Pharmacy and Research Institute of Life and Pharmaceutical Sciences, Sunchon National University, Suncheon 540-950, Republic of Korea

2 Department of Physiology and Department of Biomedical Sciences, Ajou University School of Medicine, Suwon 443-749, Republic of Korea

3 Racing Laboratory, Korea Racing Authority, Gwacheon 13822, Republic of Korea

4 Jeonbuk Branch Institute, Korea Research Institute of Bioscience and Biotechnology, Jeonbuk 56212, Republic of Korea

5 College of Pharmacy, Chosun University, Gwangju 501-759, Republic of Korea 